\title{
Zonação de recifes emersos da Área de Proteção Ambiental Costa dos Corais, Nordeste do Brasil
}

\author{
Andrea Quirino Steiner', Fernanda Maria Duarte Amaral', \\ João Renato de Barros Campos do Amaral $^{3}$, Roberto Sassi ${ }^{4} \&$ Juliana Imenis Barradas ${ }^{5}$
}

\author{
1. Departamento de Ciência Política, Universidade Federal de Pernambuco (UFPE), Rua Acadêmico Hélio Ramos, s/n, $14^{\circ}$ andar, Cidade Universitária, 50670-90, \\ Recife, PE, Brasil. (ecodea@gmail.com) \\ 2. Departamento de Biologia, Universidade Federal Rural de Pernambuco (UFRPE), Rua Dom Manoel de Medeiros, s/n, Dois Irmãos, $52171-900$ Recife, PE, Brasil. \\ (fernandaduarteamaral@gmail.com) \\ 3. Departamento de Sistemática e Ecologia, Universidade Federal da Paraíba (UFPB), Núcleo de Estudos e Pesquisas de Recursos do Mar - NEPREMAR \\ Cidade Universitária, 58059-900 João Pessoa, PB, Brasil. (sassi_rs@yahoo.com.br) \\ 4. Mestrado Profissional em Gestão do Desenvolvimento Local Sustentável, FCAP/UPE. (joaorenatoamaral@yahoo.com.br) \\ 5. Programa de Pós-graduação em Evolução e Diversidade, Universidade Federal do ABC (UFABC), Av. dos Estados, 5001, 09210-580 Santo André, SP, Brasil. \\ (julianaimenis@yahoo.com.br)
}

ABSTRACT. Emerged reef zonation of the Costa dos Corais Environmental Protection Area, northeast Brazil. The zoning reef environments allows us to evaluate possible anthropic impacts and to subsidize future management plans. This study aimed to survey the reef-flat's benthic species (with special emphasis on cnidarians) in the first line of reefs of the Costa dos Corais, as well as to calculate and compare their percentage cover. Continuous line transects were carried out in six beaches of the area, in three regions of the reef flat and with four replicates for each region. The results showed greatest cover of algae in all of the beaches except for Tamandaré, PE. Among the cnidarians, the zoanthids were predominant, but the scleractinian corals Siderastrea stellata and Favia gravida also occurred. Other organisms found were poriferans, the mollusk Brachidontes exustus and the echinoderm Echinometra lucunter. Bleaching was observed in the two species of corals found (in all of the beaches visited) and in the zoanthid Palythoa caribaeorum. Overall, the organisms recorded were characterized by their resistance to the unstable conditions of the reef-flat. Additionally, the cluster analyses showed that factors such as the nearness to bodies of water and human impact have substantial influence in the reef-flat's benthic cover composition, even more than the close distance between some areas.

KEYWORDS. Corals, transects, reef environments.

RESUMO. A zonação dos ambientes recifais permite avaliar possíveis impactos antropogênicos e subsidiar futuros planos de manejo. Este estudo teve por objetivo levantar as espécies bentônicas (com ênfase nos cnidários) no topo dos recifes emersos da Área de Proteção Ambiental (APA) Costa dos Corais, bem como calcular e comparar sua porcentagem de cobertura. Foram realizados transectos em linha contínuos, em seis praias da referida APA, em três regiões da plataforma recifal e com quatro réplicas para cada região. Os resultados mostraram maior cobertura de algas em todas as praias, com exceção de Tamandaré/PE. Entre os cnidários os zoantídeos predominaram, mas se registrou, também, a ocorrência dos corais escleractíneos Siderastrea stellata e Favia gravida. Outros organismos encontrados foram os poríferos, o molusco Brachidontes exustus e o equinodermo Echinometra lucunter. Foi observada ocorrência de branqueamento nas duas espécies de coral encontradas (em todas as praias visitadas) e no zoantídeo Palythoa caribaeorum. De forma geral, os organismos registrados se caracterizaram pela resistência às condições instáveis da plataforma recifal. Além disso, as análises de agrupamento mostraram que fatores como o aporte de rios e o impacto antrópico exercem substancial influência na caracterização da cobertura bentônica da plataforma recifal, sobrepondo-se até mesmo à proximidade geográfica de áreas.

PALAVRAS-CHAVE. Corais, transectos, ambientes recifais.

A diversidade de cnidários no Brasil não é considerada alta: segundo MigotTo et al. (1999), apenas 470 de aproximadamente 11.000 espécies conhecidas foram identificadas. No entanto, o endemismo é considerável nos corais escleractíneos: sete das quinze espécies zooxanteladas são encontradas apenas no Brasil, das quais duas ocorrem somente no litoral da Bahia (Hetzel \& CASTro, 1994). Veron (2000) questionou alguns destes endemismos, inclusive de duas espécies muito comuns no nordeste brasileiro (Siderastrea stellata Verrill, 1868 e Favia gravida Verrill, 1868); porém isso foi contestado por SANTOS et al. (2004), em comparação com congêneres do Caribe panamenho, que relatam serem estas espécies realmente válidas.

Em relação aos hidróides calcários, que também contribuem expressivamente para a edificação dos recifes, três das quatro espécies de Millepora que ocorrem no Brasil são endêmicas, entre as quais uma que ocorre apenas no Parcel do Manuel Luiz, no Maranhão: Millepora laboreli
Amaral, 2008 (Amaral et al., 2008). Formas de crescimento bastante diversas de colônias de Millepora spp. também foram relatadas no Arquipélago de Fernando de Noronha (AMARAl et al., 2002, 2008, 2009).

Segundo LeÃo \& Dominguez (2000) e Leão et al. $(2003,2006)$, quatro aspectos distinguem os recifes brasileiros da maioria dos demais: (1) a forma de crescimento inicial, em pináculos com formato de cogumelos (os chamados "chapeirões"), cujos topos podem se fundir; (2) a supracitada baixa diversidade de corais, porém com alto índice de endemismo; (3) o importante papel das algas calcárias na construção do recife e (4) a presença de sedimentos lamosos nas proximidades e/ou sobre os recifes mais próximos à costa. Além disso, o Brasil destaca-se por possuir os únicos recifes coralíneos do Atlântico Sul (PaUlay, 1997).

Collinge (2001) ressaltou que pesquisas que dão ênfase às respostas de organismos a hábitats estruturados espacialmente são úteis à conservação biológica, pois 
podem fornecer mecanismos práticos para planos de conservação e restauração de áreas. Estes estudos também podem ajudar a definir zonas de uso em reservas marinhas (RIEGL \& RIEGL, 1996). Os trabalhos de LoYA \& SLOBODKIN (1971) e LoYA (1972), além da revisão subsequente de métodos de transectos (LoYA, 1978), foram um marco no estudo quantitativo da estrutura de comunidades coralíneas. Os estudos anteriores eram quase que exclusivamente qualitativos, e os poucos quantitativos geralmente colhiam os dados de forma inadequada (LoyA, 1972). De fato, as revisões de métodos quantitativos realizadas por LoYA $(1972,1978)$ inspiraram uma série de contribuições em diferentes regiões do mundo (PICHON, 1976; MorrisseY, 1980; BouChON, 1981; Rogers et al., 1983; DusTAN \& Halas, 1987; Fenner, 1993; Carleton \& Done, 1995; RIEGL \& RiEGL, 1996, entre outros).

No Brasil, trabalhos quantitativos em comunidades coralíneas ainda são poucos, tendo sido realizados na região de Abrolhos/BA (Villaça \& Pitombo, 1997; Segal \& Castro, 2001), na Baía de Todos os Santos, BA (Ramos et al., 2010), no Atol das Rocas (GHERARDI \& BosENCE, 2001), no Arquipélago de Fernando de Noronha (Eston et al., 1986), em Arraial do Cabo/RJ (CASTRO et al., 1999) e em algumas praias do litoral pernambucano (BARRADAS et al., 2010).

A maioria dos estudos deste tipo não analisa o ambiente recifal emerso, enfocando, apenas, as porções que se encontram sempre submersas. Assim, este estudo teve como foco principal, entre os organismos bentônicos, os cnidários do topo dos recifes da Área de Proteção Ambiental (APA) Costa dos Corais. O objetivo foi analisar a composição, distribuição e porcentagem de cobertura desses organismos. Apesar da baixa diversidade da fauna coralínea dessa região, defende-se aqui a necessidade de estudá-la, pois são nestas áreas onde ocorre o contato mais direto entre o homem e o recife, em momentos de lazer (turismo) ou trabalho (pesca, coleta de organismos marinhos ornamentais) (STEINER et al., 2006). Ademais, as atividades humanas promovem a eutrofização das águas associada à poluição de esgotos domésticos e altas taxas de sedimentação e turbidez, o que se soma à ocorrência de eventos de branqueamento e doenças em corais (LEÃo et al., 2010; ANTHONY et al., 2011; MAYNARD et al., 2011).

Neste contexto, metodologias que envolvam o monitoramento de fatores naturais, juntamente com os fatores antropogênicos, são essenciais para o manejo adequado dos recursos (PIREs-VANIN et al., 2011). Assim, estudos de zonação nestas regiões podem contribuir para avaliar o impacto decorrente destas atividades e para subsidiar futuros planos de manejo.

\section{MATERIAL E MÉTODOS}

Caracterização da área. Criada por Decreto Federal em 23 de outubro de 1997, a APA Costa dos Corais abrange $135 \mathrm{~km}$ de costa, num total de 413.563 ha de áreas terrestres e marinhas, que se iniciam no Rio Formoso (município de Rio Formoso, litoral sul de Pernambuco) e vão até o Rio Meirim (município de Maceió, Alagoas). Pelo lado oeste, é limitada pela linha de preamar média (incluindo os manguezais, em toda sua extensão), e a leste, adentra o oceano por cerca de dezoito milhas náuticas, coincidindo com os limites da plataforma continental. É a maior unidade de conservação marinha do Brasil e foi criada no intuito de preservar os recifes lá existentes, antes somente protegidos em áreas fora da costa litorânea (MAIDA \& FERREIRA, 2003).

Os recifes desta área se caracterizam pelo seu crescimento isolado, em colunas com altura entre cinco e seis metros, que se expandem lateralmente no topo e se aglutinam formando grandes estruturas interligadas. A superfície destes recifes é geralmente coberta por zoantídeos e algas calcárias, além de corais como Siderastrea stellata Verrill, 1868 e Favia gravida Verrill, 1868 (MaIDA \& FERREIRA 2003).

Metodologia. A metodologia de transecto escolhida ("Continuous transect recording"; LoYA, 1978) consiste em registrar todo organismo interceptado pela linha do transecto (neste caso, mediu-se também as áreas de substrato descoberto), além de anotar a porção da linha que cobre o organismo. Apesar de a linha ser estreita, é possível estimar a cobertura dos organismos, visto que esta é proporcional à área da linha que recobre cada organismo registrado (LoYA, 1978).

Foram realizados transectos em linha contínuos durante os meses de março, novembro e dezembro de 2004, nas seguintes praias (Fig. 1): Carneiros e Tamandaré (Tamandaré, PE); Bitingui e Barreiras (Japaratinga, AL); Sonho Verde e Paripueira (Paripueira, AL). Para tanto, utilizou-se uma trena de $10 \mathrm{~m}$ que era disposta paralela à costa, em três regiões do topo recifal, na primeira linha de recifes: região mais próxima à frente recifal (região $\mathrm{A}$ ), região central da plataforma recifal (região $\mathrm{B}$ ) e região mais próxima à costa recifal (região C) (Fig. 2). Quatro réplicas foram realizadas para cada região e os organismos observados nos transectos anotados em caderneta de campo. Após verificar a normalidade dos dados com o teste de Kolmogorov-Smirnov, a porcentagem de cobertura calculada para as áreas foi avaliada utilizando análises de agrupamento (programa STATISTICA for Windows Release 4.1).

\section{RESULTADOS}

Porcentagem de cobertura. Foram encontrados nos transectos os cnidários: os corais escleractíneos Siderastrea stellata Verrill, 1868 e Favia gravida Verrill, 1868; os zoantídeos Zoanthus sp., Palythoa caribaeorum (Duchassaing \& Michelotti, 1860) e Protopalythoa variabilis (Duerden, 1898). Também foi registrada a ocorrência de algas, poríferos, o molusco bivalve Brachidontes exustus (Linnaeus, 1758), o equinodermo Echinometra lucunter (Linnaeus, 1758) e algumas ascídias não identificadas.

Diversas algas dos gêneros Sargassum, Halimeda, Caulerpa, Laurencia, Dictyota, Dictyopteris e Ulva 


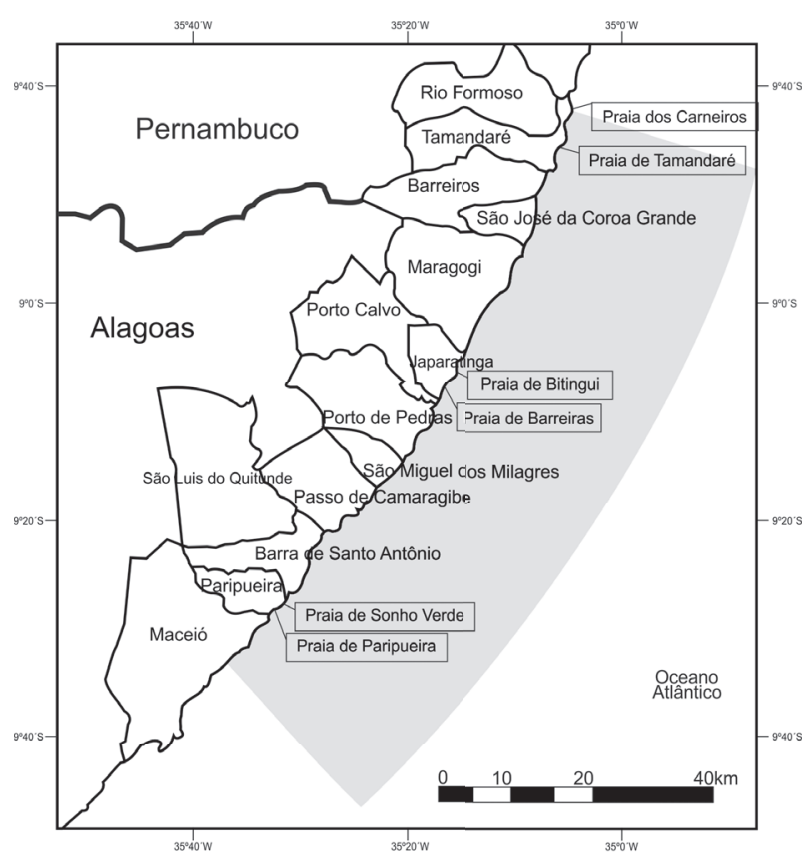

Fig. 1. Mapa dos $135 \mathrm{~km}$ de extensão litorânea da APA Costa dos Corais (em cinza), com destaque para as localidades estudadas entre março e dezembro de 2004: praias onde foram realizados estudos de porcentagem de cobertura (destacadas no retângulo) (adaptado de IBAMA, 2004).

foram registradas nos transectos examinados, porém não discriminadas em nível específico. O porífero com maior ocorrência foi Cinachyrella alloclada (Uliczka, 1929), além de Tedania ignis (Duchassaing \& Michelotti, 1864) e Haliclona sp., muito embora pela dificuldade de sua identificação em campo, as espécies desse grupo não foram distinguidas nos transectos estudados.
$\mathrm{Na}$ Praia dos Carneiros, os organismos mais abundantes foram as algas, que cobriram a maior área, com $36,2 \%$ do total (Tab. I). Esta foi a única praia onde se registrou a presença de ascídias nos transectos investigados, que cobriram $0,2 \%$ da região A, e de $B$. exustus. Salienta-se também, a extensa área de substrato descoberto $(34,4 \%$ do total), principalmente na região $\mathrm{C}$.

Em relação à composição de cnidários observada na Praia dos Carneiros, houve predominância de Siderastrea stellata $(55,3 \%$ da cobertura de cnidários) sendo mais representativo nas regiões $\mathrm{A}$ e $\mathrm{C}$, seguido por Zoanthus sp. Este último ocorreu apenas na região $\mathrm{B}$, onde representou 92,1\% dos cnidários observados.

Nos recifes da Praia de Tamandaré, os cnidários foram os organismos mais representativos (Tab. II), cobrindo $75,7 \%$ da área total, além de ser o grupo mais comum em todas as regiões. Destacaram-se também as algas, cobrindo maior área na região $\mathrm{C}$. Neste recife foram registradas poucas áreas de substrato descoberto e não foram observados poríferos, nem mesmo fora dos transectos.

Ao analisar apenas os cnidários de Tamandaré, $P$. caribaeorum foi o mais representativo, principalmente na região A. A segunda maior cobertura foi de Zoanthus sociatus (Ellis \& Solander, 1786) e P. variabilis juntos, representando a maior parte dos cnidários nas regiões B e C. Neste caso, não era possível separar as colônias, fato que só foi observado em Tamandaré e na Praia de Barreiras. Separadamente, Zoanthus sp. representou 23,2\% dos cnidários das três regiões.

As algas consistem a maior parte das observações no recife estudado em Barreiras, principalmente nas regiões $\mathrm{A}$

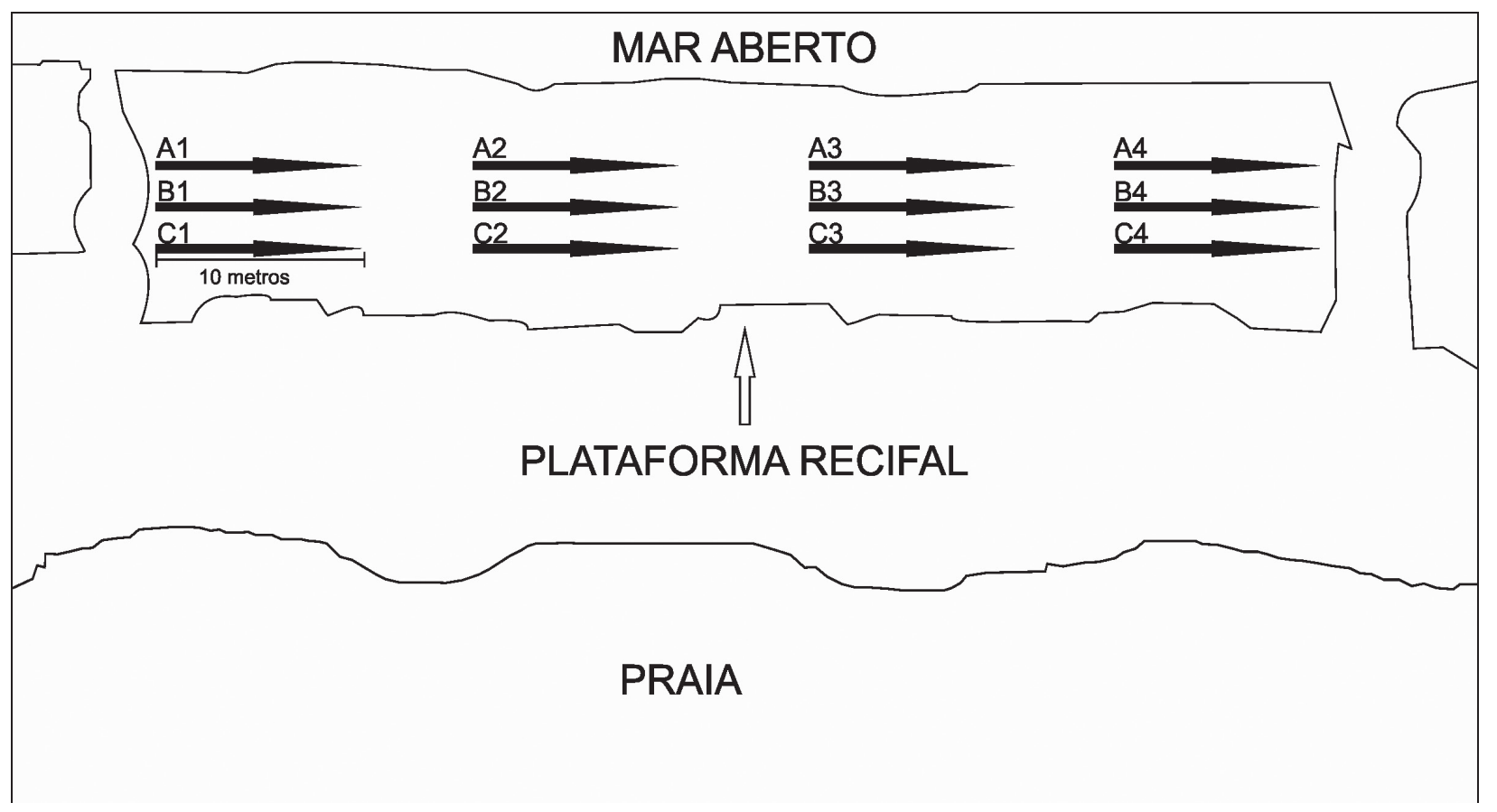

Fig. 2. Esquema dos transectos realizados nos recifes da APA Costa dos Corais, estudados entre março e dezembro de 2004; as letras representam três regiões da plataforma recifal e os números, as quatro réplicas realizadas para cada região. 
Tab. I. Porcentagem de cobertura encontrada nas três regiões avaliadas de um recife da Praia dos Carneiros, Tamandaré, PE, Brasil, em dezembro de 2004 [*, unicamente Cinachyrella alloclada (Uliczka, 1929)].

\begin{tabular}{|c|c|c|c|c|}
\hline \multirow{2}{*}{ Cobertura } & \multicolumn{4}{|c|}{ Região do recife } \\
\hline & A & B & $\mathrm{C}$ & Total \\
\hline Substrato & $11,2 \%$ & $20,8 \%$ & $71,1 \%$ & $34,4 \%$ \\
\hline Algas & $74,0 \%$ & $24,5 \%$ & $10,1 \%$ & $36,2 \%$ \\
\hline Poríferos* & $1,7 \%$ & $* 0,3 \%$ & $2,5 \%$ & $1,5 \%$ \\
\hline Cnidários & $3,5 \%$ & $15,0 \%$ & $16,3 \%$ & $11,6 \%$ \\
\hline Brachidontes exultus & $0,0 \%$ & $28,2 \%$ & $0,0 \%$ & $9,4 \%$ \\
\hline Echinometra lucunter & $9,4 \%$ & $11,2 \%$ & $0,0 \%$ & $6,9 \%$ \\
\hline Ascídias & $0,2 \%$ & $0,0 \%$ & $0,0 \%$ & $0,1 \%$ \\
\hline
\end{tabular}

Tab. II. Porcentagem de cobertura encontrada nas três regiões avaliadas de um recife da Praia de Tamandaré, Tamandaré, PE, Brasil, em dezembro de 2004.

\begin{tabular}{|c|c|c|c|c|}
\hline \multirow{2}{*}{ Cobertura } & \multicolumn{4}{|c|}{ Região do recife } \\
\hline & $\mathrm{A}$ & $\mathrm{B}$ & $\mathrm{C}$ & Total \\
\hline Substrato & $0,0 \%$ & $1,0 \%$ & $7,8 \%$ & $2,9 \%$ \\
\hline Algas & $24,0 \%$ & $7,7 \%$ & $29,5 \%$ & $20,4 \%$ \\
\hline Poríferos & - & - & - & - \\
\hline Cnidários & $76,0 \%$ & $89,4 \%$ & $61,5 \%$ & $75,7 \%$ \\
\hline Brachidontes sp. & - & - & - & - \\
\hline Echinometra lucunter & $0,0 \%$ & $1,9 \%$ & $1,2 \%$ & $1,0 \%$ \\
\hline Ascídias & - & - & - & - \\
\hline
\end{tabular}

$(89 \%)$ e B $(55,4 \%)$. Na região C, mais da metade da área total observada $(52,7 \%)$ corresponde a substrato descoberto (Tab. III).

Nesse recife, $P$. caribaeorum constituiu 100\% dos cnidários da região A e 35,2\% de todos os cnidários encontrados. Zoanthus sp. foi observado somente nas regiões B e C. Ocorreu, ainda, o conglomerado de Zoanthus sociatus e $P$. variabilis, como na Praia de Tamandaré.

$\mathrm{Na}$ Praia de Bitingui, as algas representaram 63,6\% da área total estudada no recife (Tab. IV). Também se registrou uma extensa área de substrato descoberto $(20,6 \%$ do total), principalmente na região B. Os cnidários também foram representativos (15,6\% do total). No recife de Bitingui, os cnidários só foram registrados nas regiões $\mathrm{A}$ e B, e Zoanthus sp. representou 53\% destes. Protopalythoa variabilis e $P$. caribaeorum também estiveram presentes neste recife (25,3\% e $16,5 \%$, respectivamente).

No recife da Praia de Sonho Verde, as algas foram, novamente, os organismos mais representativos (Tab. V), cobrindo $51,9 \%$ da área total estudada. No entanto, foi maioria apenas na região $\mathrm{B}$; na região $\mathrm{A}$, os cnidários se sobressaíram $(51,8 \%)$, enquanto na região $\mathrm{C}$ houve maior área de substrato descoberto $(67,6 \%)$.

Entre os cnidários de Sonho Verde, $P$. caribaeorum representou $51,7 \%$ do total da cobertura, embora apenas na região A. Nas regiões $\mathrm{B}$ e $\mathrm{C}$, foram mais representativos $P$. variabilis $(50,3 \%)$ e $S$. stellata $(82,5 \%)$, respectivamente.

$\mathrm{Na}$ Praia de Paripueira, as algas mais uma vez cobriram a maior parte da área estudada $(69,1 \%)$, sendo maioria em todas as regiões (Tab. VI). Os cnidários ocuparam apenas 5,5\% da área total e foram mais comuns na região $\mathrm{A}$. Neste recife, também se registrou uma extensa área de substrato descoberto $(21,9 \%$ do total), principalmente na região $\mathrm{B}$.

Palythoa caribaeorum, apesar de ter sido registrada somente na região A do recife de Paripueira, representou
$76,2 \%$ do total de cobertura de cnidários. Nas regiões B e C, foi observado apenas o coral S. stellata. A ocorrência dos cnidários está expressa na Tab. VII para todos os locais de estudo.

Analisando os dados como um todo, os valores de desvio padrão não evidenciam um zoneamento bem definido para os organismos estudados. A cobertura de algas, por exemplo, variou bastante entre as regiões do recife de todas as praias e não seguiu nenhum padrão. O mesmo ocorreu, em menor grau, com os cnidários. Diferentemente, os poríferos e os ouriços mantiveram-se relativamente constantes em todas as regiões dos recifes de todas as praias estudadas, indicando uma distribuição razoavelmente homogênea. Dentre os cnidários, $F$. gravida se manteve o mais constante, variando pouco em termos percentuais de região para região. Os demais variaram em diferentes graus de praia para praia, sem padrão definido.

De forma geral, constatou-se que as algas foram uma constante em todas as regiões das praias estudadas, seguidas pelos cnidários, que foram registrados em todas as localidades, com exceção da região C da Praia de Bitingui, onde ocorreram apenas algas e áreas de substrato descoberto. Dentre os corais, $S$. stellata foi o único que ocorreu em todos os recifes estudados. Favia gravida foi menos comum, ocorrendo somente em quatro praias. Em relação aos zoantídeos, as três espécies encontradas foram registradas em todas as praias. Por outro lado, nenhum zoantídeo ocorreu na região $\mathrm{C}$ dos Carneiros, região $\mathrm{C}$ de Bitingui e região $\mathrm{B}$ e $\mathrm{C}$ de Paripueira. Em relação à “associação" entre $P$. variabilis e $Z$. sociatus, esta só ocorreu em Tamandaré (regiões B e C) e Barreiras (região C).

Em relação aos demais organismos, o mais representativo foi o ouriço Echinometra lucunter, ocorrendo em todos os recifes principalmente na região B e sempre em regiões com algas. Os poríferos também ocorreram em quase todas as praias, com exceção da Praia de Tamandaré. 
Tab. III. Porcentagem de cobertura encontrada nas três regiões avaliadas de um recife da Praia de Barreiras, Japaratinga, AL, Brasil, em março de 2004.

\begin{tabular}{|c|c|c|c|c|}
\hline \multirow{2}{*}{ Cobertura } & \multicolumn{4}{|c|}{ Região do recife } \\
\hline & A & B & $\mathrm{C}$ & Total \\
\hline Substrato & $0,0 \%$ & $13,9 \%$ & $52,7 \%$ & $22,2 \%$ \\
\hline Algas & $89,0 \%$ & $55,4 \%$ & $26,1 \%$ & $56,8 \%$ \\
\hline Poríferos & $0,0 \%$ & $0,1 \%$ & $0,0 \%$ & $0,1 \%$ \\
\hline Cnidários & $11,0 \%$ & $30,1 \%$ & $18,7 \%$ & $19,9 \%$ \\
\hline Brachidontes sp. & - & - & - & - \\
\hline Echinometra lucunter & $0,0 \%$ & $0,5 \%$ & $2,5 \%$ & $1,0 \%$ \\
\hline Ascídias & - & - & - & - \\
\hline
\end{tabular}

Tab. IV. Porcentagem de cobertura encontrada nas três regiões avaliadas de um recife da Praia de Bitingui, Japaratinga, AL, Brasil, em novembro de 2004 (*, unicamente Cinachyrella alloclada).

\begin{tabular}{|c|c|c|c|c|}
\hline \multirow{2}{*}{ Cobertura } & \multicolumn{4}{|c|}{ Região do recife } \\
\hline & $\mathrm{A}$ & $\mathrm{B}$ & $\mathrm{C}$ & Total \\
\hline Substrato & $10,0 \%$ & $26,9 \%$ & $25,0 \%$ & $20,6 \%$ \\
\hline Algas & $66,7 \%$ & $49,2 \%$ & $75,0 \%$ & $63,6 \%$ \\
\hline Poríferos* & $0,0 \%$ & $0,1 \%$ & $0,0 \%$ & $0,1 \%$ \\
\hline Cnidários & $23,1 \%$ & $23,7 \%$ & $0,0 \%$ & $15,6 \%$ \\
\hline Brachidontes sp. & - & - & - & - \\
\hline Echinometra lucunter & $0,2 \%$ & $0,1 \%$ & $0,0 \%$ & $0,1 \%$ \\
\hline Ascídias & - & - & - & - \\
\hline
\end{tabular}

Tab. V. Porcentagem de cobertura encontrada nas três regiões avaliadas de um recife da Praia de Sonho Verde, Paripueira, AL, Brasil, em novembro de 2004 (*, unicamente Cinachyrella alloclada).

\begin{tabular}{lcccc}
\hline \multirow{2}{*}{ Cobertura } & \multicolumn{3}{c}{ Região do recife } \\
\cline { 2 - 5 } & $\mathrm{A}$ & $\mathrm{B}$ & $\mathrm{C}$ & Total \\
\hline Substrato & $0,0 \%$ & $8,2 \%$ & $67,6 \%$ & $25,3 \%$ \\
Algas & $48,1 \%$ & $76,6 \%$ & $31,0 \%$ & $51,9 \%$ \\
Poríferos & $0,1 \%$ & $*, 3 \%$ & $0,4 \%$ & $0,3 \%$ \\
Cnidários & $51,8 \%$ & $13,3 \%$ & $1,0 \%$ & $22,0 \%$ \\
Brachidontes exultus & - & - & - \\
Echinometra lucunter & $0,0 \%$ & $1,6 \%$ & $0,0 \%$ & $0,5 \%$ \\
Ascídias & - & - & - & - \\
\hline
\end{tabular}

Tab. VI. Porcentagem de cobertura encontrada nas três regiões avaliadas de um recife da Praia de Paripueira, Paripueira, AL, Brasil, em novembro de 2004 (*, unicamente Cinachyrella alloclada).

\begin{tabular}{lcccc}
\hline \multirow{2}{*}{ Cobertura } & \multicolumn{3}{c}{ Região do recife } \\
\cline { 2 - 5 } & $\mathrm{A}$ & $\mathrm{B}$ & $\mathrm{C}$ & Total \\
\hline Substrato & $1,7 \%$ & $42,3 \%$ & $21,6 \%$ & $21,9 \%$ \\
Algas & $83,5 \%$ & $55,6 \%$ & $68,4 \%$ & $69,1 \%$ \\
Poríferos & $* 0,1 \%$ & $* 0,5 \%$ & $3,9 \%$ & $1,5 \%$ \\
Cnidários & $14,7 \%$ & $0,6 \%$ & $1,0 \%$ & $5,5 \%$ \\
Brachidontes exultus & - & - & - & - \\
Echinometra lucunter & $0,0 \%$ & $1,0 \%$ & $5,1 \%$ & $2,0 \%$ \\
Ascídias & - & - & - & - \\
\hline
\end{tabular}

Tab. VII. Porcentagem de cobertura total de cnidários nos recifes avaliados nos seis locais de estudo.

\begin{tabular}{|c|c|c|c|c|c|c|}
\hline \multirow{2}{*}{ Cnidários } & \multicolumn{6}{|c|}{ Cobertura Total } \\
\hline & Carneiros & Tamandaré & Barreiras & Bitingui & S. Verde & Paripueira \\
\hline S. stellata & $55,3 \%$ & $0,9 \%$ & $0,5 \%$ & $5,2 \%$ & $9,3 \%$ & $17,3 \%$ \\
\hline F. gravida & $3,2 \%$ & $0,3 \%$ & $2,9 \%$ & - & $0,6 \%$ & - \\
\hline P. caribaeorum & $1,7 \%$ & $39,5 \%$ & $35,2 \%$ & $16,5 \%$ & $51,7 \%$ & $76,2 \%$ \\
\hline P. variabilis & $0,1 \%$ & $4,3 \%$ & $9,2 \%$ & $25,3 \%$ & $14,8 \%$ & $2,4 \%$ \\
\hline Zoanthus sp. & $39,7 \%$ & $23,2 \%$ & $29,7 \%$ & $53,0 \%$ & $23,6 \%$ & $4,1 \%$ \\
\hline Z. sociatus $+P$. variabilis & - & $31,8 \%$ & $22,5 \%$ & - & - & - \\
\hline
\end{tabular}

Cinachyrella alloclada foi a espécie mais comum, sendo ocorrência exclusiva em algumas áreas: regiões B dos Carneiros, Sonho Verde e Paripueira, e todas as regiões de Bitingui. Outros organismos menos representativos - ocorrendo apenas em Carneiros - foram Brachidontes exustus (região $\mathrm{B}$ ) e as ascídias (região $\mathrm{A}$ ).

Quanto às áreas de substrato descoberto, estas foram registradas em quase todas as regiões, com exceção apenas das regiões A de Tamandaré, Barreiras e Sonho Verde. Ao comparar as regiões A dos recifes estudados utilizando análise de agrupamento, pode se observar que houve maior semelhança entre Barreiras e Paripueira. Diferentemente, os recifes de Sonho Verde e Tamandaré foram mais similares em relação à composição de cnidários. 
Os recifes de Barreiras e Bitingui foram os que mais se assemelharam na região $\mathrm{B}$. Além disso, para esta área, maior semelhança foi observada entre a cobertura de cnidários de Barreiras e Tamandaré.

As regiões $\mathrm{C}$ das praias de Bitingui e Paripueira foram as mais semelhantes. Como para a região $\mathrm{B}$, a Praia de Tamandaré ficou isolada das demais. Diferentemente, a composição de cnidários das regiões $\mathrm{C}$ foi mais parecida em Paripueira e Carneiros.

Ao analisarmos todas as regiões de todos os recifes investigados, verifica-se maior semelhança entre as regiões A de Barreiras e Paripueira, seguido pelas regiões $C$ de Bitingui e Paripueira. A figura 3 mostra a formação de dois grandes grupos, um formado pelas regiões $\mathrm{C}$ dos recifes de Barreiras, Sonho Verde e Carneiros, e outro formado pelas demais regiões, com exceção da região $\mathrm{B}$ dos Carneiros, que ficou isolada.

Levando em consideração apenas os cnidários, pode-se observar a formação de dois grupos (Fig. 4), sem que nenhuma região ficasse isolada. Um grupo incluiu todas as regiões $\mathrm{A}$, com exceção de Carneiros. O outro grupo, mais próximo entre si, englobou as regiões com maior similaridade: a região $\mathrm{A}$ dos Carneiros e as regiões $\mathrm{B}$ e $\mathrm{C}$ de Paripueira, que se mostram idênticas no gráfico. Estas regiões, junto com as $\mathrm{C}$ dos Carneiros e de Sonho Verde, formaram um grupo que corresponde aos locais onde ocorreu pouca ou nenhuma cobertura de zoantídeos.

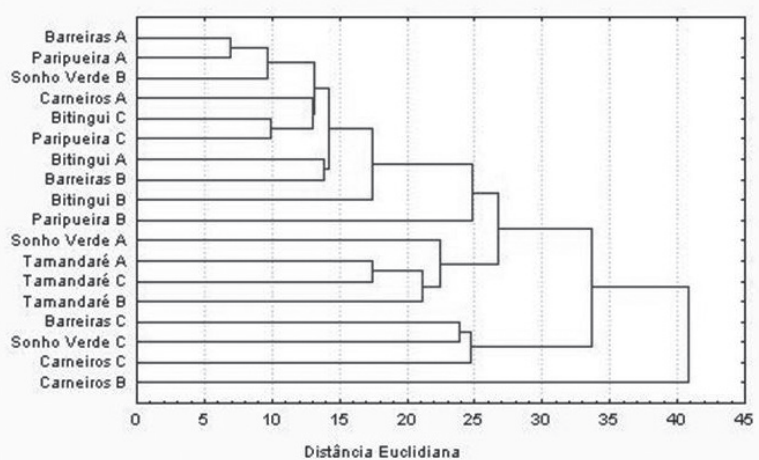

Fig. 3. Análise de agrupamento de todas as regiões dos recifes estudados na Área de Proteção Ambiental Costa dos Corais, Brasil, entre maio e dezembro de 2004

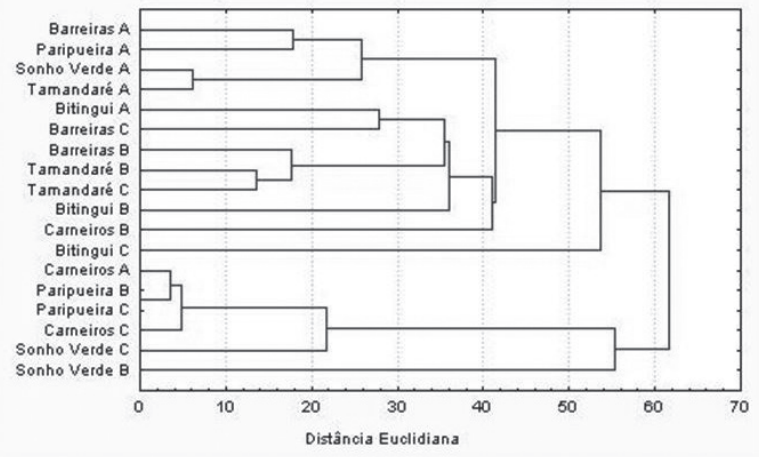

Fig. 4. Análise de agrupamento da composição de cnidários de todas as regiões dos recifes estudados na Área de Proteção Ambiental Costa dos Corais, Brasil, Brasil, entre maio e dezembro de 2004.
Ao analisarmos a soma da porcentagem de cobertura das três áreas analisadas nos recifes, pode-se observar que as praias mais similares foram Barreiras e Sonho Verde (Fig. 5). Estas formaram um grande grupo com Bitingui, Paripueira e Carneiros; mais uma vez, Tamandaré ficou isolada das demais. No entanto, em relação à composição total de cnidários (Fig. 6), Carneiros ficou isolada das demais, que formaram um grande grupo dentro do qual Barreiras e Tamandaré tiveram a maior aproximação, provavelmente devido à ocorrência conjunta de $Z$. sociatus e $P$. variabilis.

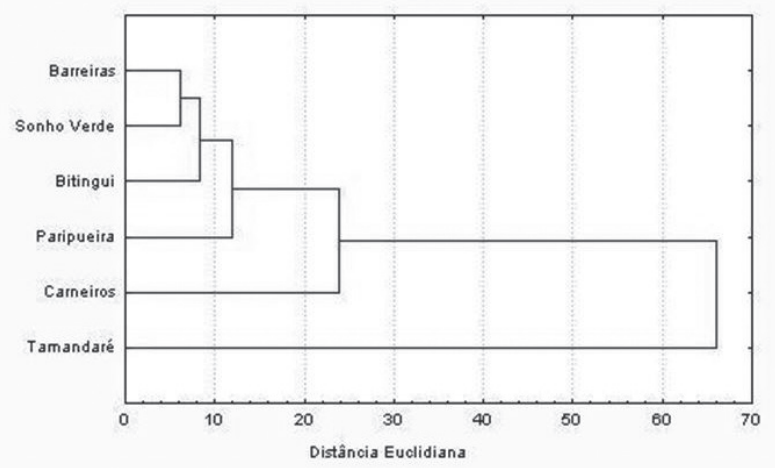

Fig. 5. Análise de agrupamento da porcentagem de cobertura total levantada em todas as regiões dos recifes estudados na Área de Proteção Ambiental Costa dos Corais, Brasil, entre maio e dezembro de 2004.

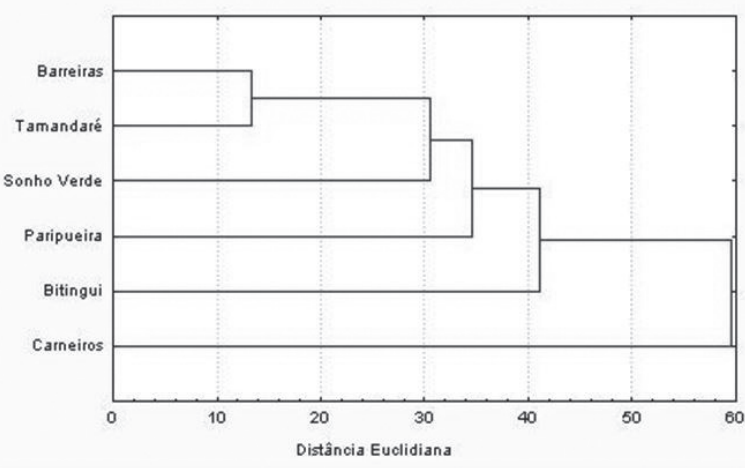

Fig. 6. Análise de agrupamento da porcentagem de cobertura de cnidários total levantada em todas as regiões dos recifes estudados na Área de Proteção Ambiental Costa dos Corais, Brasil, entre maio e dezembro de 2004

\section{DISCUSSÃO}

As algas foram predominantes em todas as praias estudadas, com exceção de Tamandaré, onde os cnidários foram maioria. Isso corrobora MoRRISSEY (1980), em estudo na Ilha Magnetic, em Queensland, Austrália e com VILLAÇA et al. (2010) para o Atol das Rocas (RN), Brasil. Este autor considerou comum espécies de Sargassum em toda a plataforma recifal estudada, bem como a alga calcária Halimeda opuntia (Linnaeus) J. V. Lamouroux, 1816, que ocorreu principalmente na porção central do recife. Fato similar foi observado por RAMOs et al. (2010) e BARRADAS et al. (2010) para a Baía de Todos os Santos (BA) e para 
a plataforma recifal da Praia de Porto de Galinhas (PE), respectivamente.

Entre os cnidários, os zoantídeos apresentaram a maior porcentagem de cobertura em todos os recifes, com exceção da Praia dos Carneiros. Segundo RoHLfS DE MACEDO \& BELÉM (1994), os zoantídeos no Brasil são muito encontrados nas zonas entremarés, sendo Zoanthus e Palythoa os gêneros mais comuns, conforme registrado neste estudo. Além disso, salienta-se a resistência das espécies encontradas: SEBENs (1982) relatou que Zoanthus sociatus e Palythoa caribaeorum resistem até nove e cinco horas à exposição direta ao sol, respectivamente. De fato, RABELO et al. (2013) demonstraram que $P$. caribaeorum e Z. sociatus são capazes de recolonizar uma área completamente exposta em poucos meses.

No entanto, em algumas regiões nenhum zoantídeo foi observado: na região $\mathrm{C}$ dos Carneiros e Bitingui e nas regiões $\mathrm{B}$ e $\mathrm{C}$ de Paripueira, áreas estas que recebem influência de cursos d'água próximos (rio Formoso, em Carneiros; rio Bitingui e rio Ilha, em Bitingui; rios Freiras e Sauaçuí, em Paripueira). Este dado corrobora Boscolo \& Silveira (2005), que afirmam que estes animais são sensíveis à poluição e a águas salobras.

Por outro lado, a maior cobertura de zoantídeos entre as regiões $\mathrm{C}$ foi registrada na Praia de Tamandaré. Levando em consideração Boscolo \& SilveIRA (2005), que enfatizaram que a fragmentação dos zoantídeos contribui para seu sucesso na dispersão e propagação de clones, a grande cobertura de zoantídeos nesta região pode estar atrelada à presença humana e seu pisoteio, visto que o recife é muito visitado por turistas, veranistas, pescadores e coletores de moluscos e crustáceos.

Entretanto, com exceção do recife da Praia dos Carneiros, a maior cobertura de $P$. caribaeorum foi sempre registrada na região $\mathrm{A}$, região mais distante $\mathrm{e}$, consequentemente, menos submetida ao pisoteio.

É muito provável que a maioria das colônias de Zoanthus observadas fosse Zoanthus sociatus (Ellis \& Solander, 1786). Porém, dada a dificuldade de distinguir as espécies deste gênero em campo e a impossibilidade de realizar coleta devido à condição de Área de Proteção Ambiental do local, neste trabalho optou-se por se referir a este organismo como Zoanthus sp. Abre-se uma exceção, entretanto, para o caso da ocorrência "conjunta" de Z. ociatus e $P$. variabilis (Duerden, 1898), por ser um fenômeno registrado na literatura (Sebens, 1982).

Apesar de sua proximidade com a foz do rio Formoso e possíveis sedimentos, a Praia dos Carneiros foi a única em que se registrou todas as espécies levantadas nos transectos (inclusive a maior cobertura de cnidários, principalmente corais). Além disso, foi a única praia onde se registrou Brachidontes exustus e ascídias e, por outro lado, foi onde houve maior área de substrato descoberto; porém estas áreas ocorreram principalmente na região $\mathrm{C}$, que recebe o aporte de água doce do rio Formoso.

$A$ região $\mathrm{C}$ de Bitingui foi a única onde não ocorreu cnidários. Salientam-se as condições especiais deste recife: (1) elevada quantidade de sedimento arenoso, principalmente nas regiões $\mathrm{B}$ e $\mathrm{C}$; (2) recife extremamente largo (aproximadamente $1.000 \mathrm{~m}$ ), no qual a quantidade de poças diminui expressivamente na direção do supralitoral; (3) localizado em uma região irrigada por vários riachos, que juntos podem influenciar a salinidade e turbidez do local. Assim, é possível que, juntas, estas condições possam influir na ocorrência de cnidários.

Segundo Loya (1972), o topo recifal de recifes emersos ou parcialmente emersos é uma zona instável, severa e imprevisível em relação às áreas mais profundas do recife. Por estes motivos, este autor acredita que a plataforma recifal seja mais pobre em diversidade. Isto explicaria o porquê de apenas Favia gravida e Siderastrea stellata nos recifes estudados. Por outro lado, esse fato aponta para a resistência destas duas espécies, ideais às condições físicas extremamente mutáveis da plataforma recifal, que incluem grandes variações de temperatura, salinidade e ação de ondas (SANDERS, 1969 apud LOYA, 1972). Porém, registrou-se menor cobertura de $F$. gravida que de $S$. stellata, além da primeira espécie só ter ocorrido em quatro das seis praias estudadas (sempre em locais com ocorrência de S. stellata).

Siderastrea stellata não ocorreu em algumas regiões de três dos recifes estudados: região A de Tamandaré, regiões $\mathrm{A}$ e $\mathrm{B}$ de Barreiras e região $\mathrm{C}$ de Bitingui. Como discutido anteriormente, a região $\mathrm{C}$ de Bitingui não apresentou nenhum cnidário. Motivos similares podem explicar a não ocorrência deste coral em Barreiras, uma praia adjacente a Bitingui. No entanto, a situação em Barreiras é um pouco diferente, pois o recife estudado é cortado por um canal principal e vários secundários, que fornecem hábitat mais favorável durante a maré baixa devido ao acúmulo de água e consequente proteção contra a dissecação. Assim, é provável que a região $\mathrm{C}$ de Barreiras, por ser mais próxima a estes canais, tenha oferecido melhores condições ao crescimento de $S$. stellata.

O porífero mais encontrado foi Cinachyrella alloclada, o que corrobora com Esteves et al. (2002), que descreveram esta espécie como de ampla faixa de distribuição, ocorrendo fixa a substrato de arenito, desde as zonas mais expostas ao impacto das ondas, até regiões protegidas.

Em relação ao ouriço Echinometra lucunter, este foi encontrado em todas as praias, principalmente na região $\mathrm{B}$ dos recifes.

Ao analisar os dados como um todo, nenhum padrão de zoneamento para os organismos ficou evidente no topo dos recifes estudados; de forma geral, os resultados dos transectos e as análises de agrupamento sugerem variação devido às características locais de cada habitat.

Mudanças morfológicas e no padrão de distribuição de espécies podem ser explicadas também pelas variações na temperatura global que vêm ocorrendo ao longo dos anos (Clemente et al., 2011; Mora \& SAle, 2011). Ademais, o estudo mostrou que os topos dos recifes estudados se caracterizam pela baixa diversidade, composta por espécies 
resistentes às condições instáveis e severas desta região recifal e comuns na costa nordeste brasileira.

\section{REFERÊNCIAS BIBLIOGRÁFICAS}

Amaral, F. D.; Broadhurst, M. K.; Cairns, S. D. \& Schlenz, E. 2002. Skeletal morphometry of Millepora occurring in Brazil, including a previously undescribed species. Proceedings of Biology of the Washington Society 115(3):681-695.

Amaral, F. M. D.; Steiner, A. Q.; Broadhurst, M. \& Cairns, S. D. 2008. An overview of the calcified hydroids from Brazil, including a new species. Zootaxa 1930:56-68.

Amaral, F. M. D.; Ramos, C. A. C.; Leão, Z. M. A. N.; Kikuchi, R. K. P.; Lima, K. K. M.; Longo, L. L.; Cordeiro, R. S.; Lira, S. M. A. \& VASCONCELOS, S. L. 2009. Checklist and morphometry of benthic cnidarians from the Fernando de Noronha Archipelago, Pernambuco, Brazil. Cahiers de Biologie Marine 50:277-290.

Anthony, K. R. N.; Maynard, J. A.; Diaz-Pulido, G.; Mumby, P. J.; Marshall, P. A. \& Hoegh-Guldberg, L. O. 2011. Ocean acidification and warming will lower coral reef resilience. Global Change Biology 17:1798-1808.

Barradas, J. I.; Amaral, F. D.; Hernández, M. I. M.; FloresMontes, M. J. \& Steiner, A. Q. 2010. Spatial distribution of benthic macroorganisms on reef flats at Porto de Galinhas Beach (northeastern Brazil), with special focus on corals and calcified hydroids. Biotemas 23(2):61-67.

Boscolo, H. K. \& Silveira, F. L. 2005. Reproductive biology of Palythoa caribaeorum and Protopalythoa variabilis (Cnidaria, Anthozoa, Zoanthidea) from the southeastern coast of Brazil. Brazilian Journal of Biology 65:29-41

Bouchon, C. 1981. Quantitative study of the Scleractinian Coral Communities of a Fringing Reef of Reunion Island (Indian Ocean). Marine Ecology 4:273-288.

Carleton, J. H. \& Done, T. 1995. Quantitative video sampling of coral reef benthos: large-scale application. Coral Reefs 14:35-46.

Castro, C. B.; Echeverría, C. A.; Pires, D. O. \& Fonseca, C. G. 1999. Distribuição do bentos (Cnidaria e Echinodermata) em costões rochosos da Baía de Ilha Grande, Rio de Janeiro, Brasil. In: Silva, S. H. G. \& LaVrado, H. P. eds. Ecologia dos Ambientes Costeiros do Estado do Rio de Janeiro. Rio de Janeiro, PPGE-UFRJ, v. 7, p.179-193.

Clemente, S.; Rodríguez, A.; Brito, A.; Ramos, A.; Monterroso, O. \& HeRNÁNDEZ, J. C. 2011. On the occurrence of the hydrocoral Millepora (Hydrozoa: Milleporidae) in the subtropical eastern Atlantic (Canary Islands): is the colonization related to climatic events? Coral Reefs 30:237-240.

Collinge, S. K. 2001. Spatial ecology and biological conservation. Biology Conservation 100:1-2.

Dustan, P. \& Halas, J. C. 1987. Changes in the reef community of Carysfort Reef, Key Largo, Florida: 1974 to 1982. Coral Reefs 6:91-106.

Esteves, E. L.; Moraes, F.; Muricy, G. \& Amaral, F. M. D. 2002. Duas novas ocorrências da Ordem Hadromerida (Porifera, Demospongiae) para o Arquipélago de São Pedro e São Paulo, Brasil. Boletim do Museu Nacional 488:1-12.

Eston, R.; Migotto, A. E.; Oliveira, F. E. C.; Rodrigues, A. S. \& Freitas, J. C. 1986. Vertical Distribution of Benthic Marine Organisms on Rocks Coasts of the Fernando de Noronha Archipelago (Brasil). Boletim do Instituto de Oceanografia 34:37-53.

FENNER, D. P. 1993. Some reefs and corals of Roatan (Honduras), Cayman Brac, and Little Cayman. Atol Reseach Bulletin (388):1-30.

Gherardi, D. F. M. \& Bosence, D. W. J. 2001. Composition and community structure of the coralline algal reefs from Atol das Rocas, South Atlantic, Brazil. Coral Reefs 19:205-219.

Hetzel, B. \& Castro, C. B. 1994. Corais do Sul da Bahia. São Paulo, Nova Fronteira. 189p.

Leão, Z. M. A. N. \& Dominguez, J. M. L. 2000. Tropical Coast of Brazil. Marine Pollution Bulletin 41(1-6):112-122.

Leão, Z. M. A. N.; Dutra, L. X. C. \& Spanó, S. 2006. A rapid biodiversity assessment of the Abrolhos Bank: the characteristics of bottom sediments. Bulletin of Biological Assessment 38:75-81.
LEÃo, Z. M. A. N.; KIKUCHI, R. K. P. \& TeSTA, V. 2003. Corals and coral reefs of Brazil. In: CoRTÉs, J. ed. Latin American Coral Reefs. Amsterdam, Elsevier, p. 9-52.

LEÃo, Z. M. A. N.; KIKUCHI, R. P. K.; OliveIRA, M. D. M. \& VASCONCElos, V. 2010. Status of Eastern Brazilian coral reefs in time of climate changes. Pan-American Journal of Aquatic Science 5(2):224-235.

LoYA, Y. 1972. Hermatypic corals at Eilat, Red Sea. Marine Biology 13(2):100-123.

1978. Plotless and transect methods. In: StodDaRT, D. R. \& Johannes, R. F. eds. Coral reefs: research methods. Paris, United Nations, p. 197-217.

Loya, Y. \& SLOBodkin, L. H. 1971. The coral reefs of Eilat (Gulf of Eilat, Red Sea). Symposium of Zoology of the London Society (28):117-139.

Maida, M. \& Ferreira, B. P. 2003. Área de Proteção Ambiental Costa dos Corais. In: Prates, A. P. L. ed. Atlas dos Recifes de Coral nas Unidades de Conservação Brasileiras. Brasília, Ministério do Meio Ambiente, p. 86-90.

Maynard, J. A.; TURner, P. J.; Anthony, K. R. N.; Baird, A. H.; Berkelmans, R.; Eakin, C. M.; Johnson, J.; Marshall, P. A.; PACKer, G. R.; ReA, A. \& Willis, B. L. 2011. Reef Temp: an interactive monitoring system for coral bleaching using high-resolution SST and improved stress predictors. Geophysical Research Letters 35:5.

Migotto, A. E.; Silveira, F. L.; SchlenZ, E. \& Freitas, J. C. 1999. Filo Cnidaria. In: Migotto, A. E. \& Tiago, C. G. eds. Biodiversidade do Estado de São Paulo - Síntese do conhecimento ao final do Século XX. São Sebastião, USP, v. 3, p. 36-46.

Mora, C. \& Sale, P. F. 2011. Ongoing Global Biodiversity Loss and the Need to Move Beyond Protected Areas: A Review of the Technical and Practical Shortcomings of Protected Areas on Land and Sea. Marine Ecology Progress Series 434:251-266.

MorrisSEY, J. 1980. Community structure and zonation of macroalgae and hermatypic corals on a fringing reef flat of Magnetic Island (Queensland, Australia). Aquatica Botanica 8:91-139.

Paulay, G. 1997. Diversity and distribution of reef organisms. In: Birkeland, C. ed. Life and death of coral reefs. New York, Chapman \& Hall, p. 298-353.

Pichon, M. 1976. Comparative Analysis of Coral Reef Community Structures in the Vicinity of Lizard Island (Australia). National Geographic Society Research Reports - 1976 Projects, p. 711-719.

Pires-Vanin, A. M. S.; Muniz, P.; De LeO, F. C. 2011. Benthic Macrofauna Structure in the Northeast Area of Todos os Santos Bay, Bahia State, Brazil: Patterns on Spatial and Seasonal Distribution. Brazilian Journal of Oceanography 59(1):27-42.

Rabelo, E. F.; Soares, M. O. \& Cascon, H. M. 2013. Competitive Interactions Among Zoanthids (Cnidaria: Zoanthidae) in an Intertidal Zone of Northeastern Brazil. Brazilian Journal of Oceanography 61(1):35-42.

Ramos, C. A. C.; Amaral, F. D.; Kikuchi, R. K. P.; Chaves, E. M. \& MeLo, G. R. 2010. Quantification of reef benthos communities and variability inherent to the monitoring using video transect method. Environmental Monitoring and Assessment 162:95-101.

RIEGL, B. \& RiEgL, A. 1996. Studies on coral community structure and damage as a basis for zoning marine reserves. Biology Conservation 77:269-277.

Rogers, C. S.; Gilnack, M. \& Fitz III, H. C. 1983. Monitoring of coral reefs with linear transects: a study of storm damage. Journal of Experimental Marine Biology and Ecology 66:285-300.

Rohlfs de Macedo, C. M. R. \& Belém, M. J. C. 1994. The genus Zoanthus in Brazil. Characterization and Anatomical revision of Zoanthus sociatus (Cnidaria, Zoantharia, Zoanthidae). Iheringia, Série Zoologia (77):135-144

Santos, M. G.; Amaral, F. D.; Hernández, M. I. M.; Knowlton, N. \& JARÁ, J. 2004. Variação morfológica de Favia gravida Verrill, 1868 e Siderastrea stellata Verrill, 1868 (Cnidaria, Scleractinia): aspectos esqueléticos. Boletim do Museu Nacional (517):1-9.

SEBENS, K. P. 1982 Intertidal distribution of zoanthids on the Caribbean coast of Panama: effects of predation and desiccation. Bulletin of Marine Science 32(1):316-335.

Segal, B. \& Castro, C. B. 2001. A proposed method for coral cover assessment: a case study in Abrolhos, Brazil. Bulletin of Marine Science 69(2):487-496. 
Steiner, A. Q.; Eloy, C. C.; Amaral, J. R. B. C.; Amaral, F. D. \& Sassi, R. 2006. O turismo em áreas de recifes de coral: considerações acerca da Área de Proteção Ambiental Costa dos Corais (Estados de Pernambuco e Alagoas). OLAM - Ciência e Tecnologia 6(2):281296.

Veron, J. E. N. 2000. Corals of the World. Townsville, AIMS. 490p.
Villaça, R. \& Pitombo, F. B. 1997. Benthic communities of shallowwater reefs of Abrolhos, Brazil. Revista Brasileira de Oceanografia 45(1/2):35-43.

Villaça, R. C.; Fonseca, A. C.; Jensen, V. K. \& Knoppers, B. 2010. Species composition and distribution of macroalgae on Atol das Rocas, Brazil, SW-Atlantic. Botanica Marina 53:113-122. 